\title{
Wave-Like Properties of Time
}

\author{
$\underline{\text { Goutam Kumar Chandra }}{ }^{1}$ \\ ${ }^{1}$ Department of Electronics and Telecommunication Engineering,Indian \\ Institute of Engineering Science and Technology, Shibpur \\ Howrah, West Bengal, 711103 , India \\ *chandratphysics@ rediffmail.com
}

\begin{abstract}
Time is a component quantity of various measurements and is also used on its own to sequence events, to compare the duration of events or the intervals between them and to quantify rates of change in quantities in material reality or in the conscious experience [1].The equipment for measuring time might well be a clock. By making a presupposition that a clock is time, it is perceived experimentally that "time exhibits wave-like properties". Keywords: Tools and Metrology, Time and Frequency, Philosophy of Science, Special Relativity, Semiconductors
\end{abstract}

PACS Nos: 06.20.-f, 06.30.Ft , 01.70.+w ,03.30.+p , 61.82.Fk

\section{Introduction}

Time has been a perplexing quantity since Galileo's time, and it plays a predominant role in science, philosophy, and religion. Many philosophical, metaphysical and physical interpretations of time have been introduced by Galileo, Newton, Einstein and other philosophers [1-2]. Space was considered a Euclidean element since the time of Newton. Einstein replaced Newtonian laws of motion in 1905 in his theory of special relativity, where the speed of light in vacuum has the same value of $299,792,458 \mathrm{~m} / \mathrm{s}$ irrespective of the reference frame. This 1905 theory also unified space and time with the so-called space-time continuum, where time is a coordinate time and 
complex. What is known to date is that proper time is what an ideal clock measures[3]. In this paper, I have tried to give a unified understanding of time and space-time based on experiment. However, to do so, I must construe the Michelson-Morley experiment [4] as proving the postulate of special relativity.

\section{Experimental Setup}

An experiment was performed by synchronizing two digital clocks at the same time, which can measure every second precisely. Both digital clocks are electronically displayable in the 'hh: mm: ss' time format using an LCD. The clock pieces are built using the same technology and are similar in structure. The digital clock exhibits the ill effect of timing jitter[5].Both clocks navigated different paths compared to each other over the duration of ten days, and $\Delta \mathrm{t}$ was measured continually within some finite time interval at the observed reference frame $O$, where $\Delta \mathrm{t}$ is the time difference between two clocks at a certain point in time. The moving digital clock acted as a space-time odometer. The environmental conditions were similar to those for both clocks during the ten days. Let us represent two clocks as two additional reference frames. Let the first clock be called $S$ and the other be called $S^{\prime} . S$ has a coordinate $\{x, y, z, t\}$ within $S$, and $S^{\prime}$ has a coordinate $\left\{x^{\prime}, y^{\prime}, z^{\prime}, t^{\prime}\right\}$ within $S^{\prime} . S$ and $S^{\prime}$ move in uniform relative motion (velocity $v$ ) along the common direction of $x$ and $x^{\prime}$. The clock synchronization was carried out at time $t^{\prime}=t=$ 0. Initially, $S^{\prime}$ was kept 3 seconds ahead of $S$,so $\Delta \mathrm{t}=3$. The velocity of $S^{\prime}$ was greater than the velocity of $S$, but the relative velocity between $S^{\prime}$ and $S, v \ll c$, where $c$ is the speed of light in vacuum. $\Delta \mathrm{t}$ was determined to be a nondetermining wave-like function over time.

\section{Supporting Mathematics}

$f_{1}(t)$ and $f_{2}(\mathrm{t})$ are the corresponding time functions for $S^{\prime}$ and $S$

$$
f_{1}(t)-f_{2}(t)=\Delta t
$$

$\Delta t$ is a time reliant on the wave-like function shown in Fig.1. 
Lemma:if $\Delta \mathrm{t}$ is a time reliant on a wave-like function, $f_{1}(t)$ and $f_{2}(t)$ similarly are wave-like functions.

If the clock measures time, we have the following set of equations:

$$
\begin{gathered}
x^{\prime}=x-v t \\
y^{\prime}=y \\
z^{\prime}=z \\
t^{\prime}=t=\text { wave-like function }
\end{gathered}
$$

Therefore, Minkowskian $\mathbb{R}^{4}$ flat space-time can be deduced from

$$
d s^{2}=-d f(t)^{2}+d x^{2}+d y^{2}+d z^{2}
$$

where $f(t)$ is a wave-like time function.

If the clock does not measure time, we have the following set of equations:

$$
\begin{gathered}
x^{\prime}=x-v t \\
y^{\prime}=y \\
z^{\prime}=z \\
t^{\prime}=t=0
\end{gathered}
$$

Therefore, Minkowskian $\mathbb{R}^{4}$ flat space-time can be deduced from

$$
d s^{2}=d x^{2}+d y^{2}+d z^{2}
$$

which is a Euclidian $\mathbb{R}^{3}$ space.

\section{Conclusion}

Can we determine the time manifesting wave-

like behavior? 


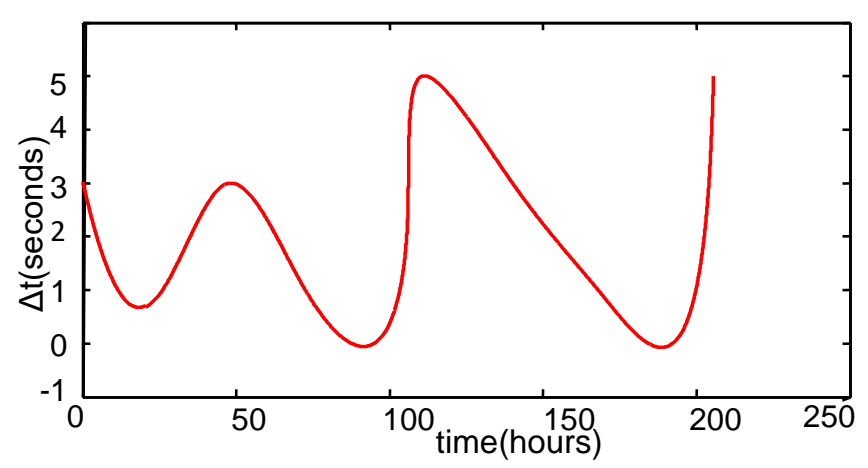

Fig. 1 Because of the pll jitter component, the moving digital clock goes both slow and fast

\section{References:}

[1] Le Poidevin, R. The experience and perception of time. In: Zalta E.N. (ed.) The Stanford Encyclopedia of Philosophy (2011).

https://plato.stanford.edu/archives/win2004/entries/time-experience/

[2]Einstein, A. Zur Elektrodynamik bewegter Körper. Ann. Phys. 322,891-921(1905).

[3]Ashby, N. Relativity in the Global Positioning System. Living Rev. Relativ. 6,1-42(2003).

[4] Michelson, A.A., Morley, E.W.On the Relative Motion of the Earth and the Luminiferous Ether. Am. J. Sci. 34, 333-345(1887).

[5] Lee, D.C. Analysis of jitter in phase-locked loops. IEEE Trans. Circuits Syst. II Analog Digit. Signal Process. 49,704-711(2002). 